\title{
Response to Chan
}

\author{
Ji Hyun $\mathrm{Kim}^{1,2}$ (1) Yo Han Ahn ${ }^{2,3,4}$ (])
}

Received: 30 December 2021 / Revised: 3 January 2022 / Accepted: 4 January 2022 / Published online: 3 February 2022

(c) The Author(s), under exclusive licence to International Pediatric Nephrology Association 2022

\section{Dear Editors,}

We thank Dr. Chan for the valuable comments in response to our recent article in Pediatric Nephrology regarding risk factors for a urinary tract infection (UTI) by extended-spectrum beta-lactamase (ESBL)-positive bacteria [1]. As a response to the letter to the editor from Dr. Chan, we want to make some comments.

Although its prevalence varies from country to country, from 10 to $40 \%$, the proportion of ESBL-positive UTIs is increasing worldwide in general over time. This could be related to an increase in the use of broad-spectrum antibiotics. Park et al. showed a linear increase in the prevalence of UTIs by ESBL-positive organisms in regions where thirdgeneration cephalosporines were highly prescribed [2]. Continuous exposure to antibiotics places selective pressure on individual pathogens, resulting in an increase in the durability and reproduction of multidrug-resistant bacteria, which either directly or indirectly increase the risk of infection by resistant strains. These bacteria may be transmitted to previously unexposed children through the environment or via contact with individuals who have already been colonized. Therefore, if the prescription rate of broad-spectrum antibiotics is higher in mainland China than in Hong Kong, this could account for a higher prevalence of infections by resistant bacteria in China than in Hong Kong.

To some extent, we agree with Dr. Chan's perspective on continuing the use of empirical antibiotics for

Yo Han Ahn

yhahn@snu.ac.kr

1 Department of Pediatrics, Seoul National University Bundang Hospital, Seongnam, Republic of Korea

2 Department of Pediatrics, Seoul National University College of Medicine, Seoul, Republic of Korea

3 Department of Pediatrics, Seoul National University Children's Hospital, 101 Daehak-ro, Jongno-gu, Seoul 03080, Republic of Korea

4 Kidney Research Institute, Medical Research Center, Seoul National University College of Medicine, Seoul, Republic of Korea treating children with ESBL-positive UTIs based on clinical response rather than in vitro susceptibility. Hyun et al. reported that among ESBL-positive UTI cases treated with non-susceptible empirical antibiotics, $89 \%$ of the cases demonstrated a good clinical response and 55\% maintained the non-susceptible antibiotics at the end of treatment [3]. In this study, the relapse and scarring rates were not significantly different between children with ESBL-positive UTIs who were treated with susceptible antibiotics vs. those treated with non-susceptible but clinically effective antibiotics. However, evidence based solely on clinical response is insufficient for forming a consensus on the adequacy of antibiotic use, regardless of in vitro susceptibility. Further studies are needed to determine the appropriate use of antibiotics in children with ESBL-positive UTIs.

\section{Declarations}

Conflict of interest The authors declare no competing interests.

\section{References}

1. Kim JH, Lee J, Kim DH, Park JY, Lee H, Kang HG, Ahn YH (2022) Maternal antibiotic exposure during pregnancy is a risk factor for community-acquired urinary tract infection caused by extended-spectrum beta-lactamase-producing bacteria in infants. Pediatr Nephrol 37:163-170

2. Park JY, Kang HM, Kwak EM, Rhim J-W, Ahn YH, Lee H, Jeong DC, Kang JH (2020) Impact of antibiotic prescribing patterns on susceptibilities of uropathogens in children below 24 months old. Antibiotics (Basel) 9:915

3. Hyun HS, Kim JH, Cho MH, Park E, Ha I-S, Cheong HI, Kang HG (2019) Low relapse rate of urinary tract infections from extended-spectrum beta-lactamase-producing bacteria in young children. Pediatr Nephrol 34:2399-2407

Publisher's note Springer Nature remains neutral with regard to jurisdictional claims in published maps and institutional affiliations. 